Preprint E2-94-484

December 1994

\title{
Temperature and Entropy of a Quantum Black Hole and Conformal Anomaly
}

\author{
Dmitri V. Fursaev' \\ Bogoliubov Laboratory of Theoretical Physics, \\ Joint Institute for Nuclear Research, \\ 141 980, Dubna, Moscow Region, Russia
}

\begin{abstract}
Attention is paid to the fact that temperature of a classical black hole can be derived from the extremality condition of its free energy with respect to variation of the mass of a hole. For a quantum Schwarzschild black hole evaporating massless particles the same condition is shown to result in the following one-loop temperature $T=(8 \pi M)^{-1}\left(1+\sigma\left(8 \pi M^{2}\right)^{-1}\right)$ and entropy $S=4 \pi M^{2}-\sigma \log M$ expressed in terms of the effective mass $M$ of a hole together with its radiation and the integral of the conformal anomaly $\sigma$ that depends on the field species. Thus, in the given case quantum corrections to $T$ and $S$ turn out to be completely provided by the anomaly. When it is absent $(\sigma=0)$, which happens in a number of supersymmetric models, the one-loop expressions of $T$ and $S$ preserve the classical form. On the other hand, if the anomaly is negative $(\sigma<0)$ an evaporating quantum hole seems to cease to heat up when its mass reaches the Planck scales.
\end{abstract}

PACS number(s): 04.60.+n, 12.25.+e, 97.60.Lf, 11.10.Gh

\footnotetext{
${ }^{1}$ e-mail: fursaev@theor.jinrc.dubna.su
} 
Black hole thermodynamics is known to possess a number of puzzles like the meaning of black hole entropy, the information loss problem and the operation of the generalized second law [1]. The principal difficulty on the way to their resolution is the lack of a consistent theory of quantum gravity. Even investigation of quantum effects on the classical curved backgrounds sometime represents a technical problem where results can be obtained only approximately. This is a reason why exactly solvable two-dimensional models of black holes are of great interest at the present moment [2].

The aim of this paper is to show how the one-loop corrections to the temperature and entropy of the 4-dimensional Schwarzschild black hole with massless quantum fields can be derived explicitly in a simple thermodynamical treatment based on the scaling properties of the theory.

To begin with, we remind that the energy $E$ and entropy $S$ of a canonical ensemble at the temperature $\beta^{-1}$ can be derived from the free energy $F(\beta)$ as follows:

$$
E=\frac{\partial}{\partial \beta}(\beta F) \quad, \quad S=\beta(E-F) .
$$

These quantities for a system being at the fixed temperature change until a system reaches a thermal equilibrium characterized by a minimum of $F[3]$. In this state the condition of extremum for $F$

$$
(\delta F)_{\beta}=0
$$

gives a relation between $\beta$ and other parameters of the ensemble. Moreover, the first law of thermodynamics in its simplest form

$$
\beta^{-1} \delta S=\delta E
$$

turns out to be a consequence of (四) and (2).

Now, returning to thermodynamics of black holes, an extremality condition of $F$, similar to (2), can be used to relate the temperature of the hole with its other parameters (mass, charge, etc.). To see this, we make use of the Gibbons-Hawking approach to gravitational thermodynamics 四. In its framework the free energy in the semiclassical approximation is given by the Euclidean Einstein-Hilbert action $W_{c l}$ with suitably 
subtracted boundary terms [

$$
\beta F(\beta)=W_{c l}(\beta)=-\frac{1}{16 \pi}\left(\int R \sqrt{g} d^{4} x+2 \int\left(K-K_{0}\right) \sqrt{h} d^{3} x\right)
$$

This functional is taken on the corresponding gravitational instanton. To elucidate the idea, consider as an example the Schwarzschild black hole with the mass $m$. The Euclidean metric reads

$$
d s^{2}=\left(1-\frac{2 m}{r}\right) d \tau^{2}+\left(1-\frac{2 m}{r}\right)^{-1} d r^{2}+r^{2} d \Omega^{2}
$$

and the presence of the temperature $\beta^{-1}$ implies the periodicity of this solution in $\tau$

$$
0 \leq \tau \leq \beta
$$

Although at arbitrary $\beta$ and $m$ the space (5) has a conical singularity at the horizon $r=2 m$, the integral curvature in (đ) on such a space is well-defined and it is non-zero. One can show [5], [6] that on (5)

$$
\int R \sqrt{g} d^{4} x=4 \pi\left(1-\frac{\beta}{8 \pi m}\right) A
$$

where $A=16 \pi m^{2}$ is the area of the horizon. Plugging (7) in (4) and taking into account the boundary terms, we get the free energy

$$
F(\beta, m)=m-4 \pi m^{2} \beta^{-1}
$$

The definitions (四) applied to (8) show that the energy of the system equals the mass of the black hole, whereas its entropy is given by the Bekenstein-Hawking formula

$$
E=m \quad, \quad S=\frac{1}{4} A
$$

Finally, finding the extremum of (8) at fixed $\beta$

$$
\frac{\partial F(\beta, m)}{\partial m}=1-8 \pi m \beta^{-1}=0
$$

one can gets the desired relation $\beta^{-1}=(8 \pi m)^{-1}$ between the temperature of the Hawking radiation and the mass of the hole. However, as distinct from a normal canonical ensemble, a black hole is the maximum of $F(\beta, m)$ rather than the minimum, which indicates its well-known instability due to evaporation.

\footnotetext{
${ }^{2}$ For simplicity we use the system of units $\hbar=c=G=k_{B}=1$.
} 
For simplicity we deal with the Schwarzschild black holes but one can show that an analogous consideration for charged holes or those in a cavity of a finite size is also possible. In particular, in these cases the value of the Hawking temperature can also be obtained in the same manner from the extremum of $F$ with respect to variation of the mass of the hole when other parameters are fixed. This fact is not surprising. Indeed, even if the gravitational action (舟) is considered on a class of manifolds admitting conical singularities, its extrema do not change and they correspond to the smooth geometries [5], [6]. A physical reason for this is the absence of such a matter distribution over the horizon which could give rise to conical defects. On the other hand, different masses $m$ under fixed $\beta$ are equivalent to Euclidean black holes with different ranging of the time coordinate $\tau$, and their free energy has the extremum when the conical singularity vanishes, which is usually associated with the Hawking temperature [4].

Consider now the black-hole thermodynamics with the one-loop quantum corrections. We will be interested in the Schwarzschild hole evaporating massless particles, so far as in this case quantum effects can be evaluated explicitly. In quantum theory the effective action and the free energy read

$$
\beta F(\beta)=W(\beta)=W_{c l}(\beta)+W_{Q}(\beta)
$$

Here $W_{c l}$ is the classical action (4) and $W_{Q}$ is a one-loop contribution to it from $N_{0}$ scalar fields and, possibly, from other fields of the higher spins (h.s.), which depends on the model in question,

$$
W_{Q}=\frac{N_{0}}{2} \log \operatorname{det} \nabla_{\mu} \nabla^{\mu}+h . s .
$$

computed on the background space (每).

Several remarks concerning (11) and (12) are in order. To get rid off the standard ultraviolet divergences in $W_{Q}$, one should include in (11) higher order curvature terms. This also enables one to remove completely [7] the additional divergences in the entropy of a black hole that are concentrated on its horizon [8]. However, for the case of the Schwarzschild hole the role of $R^{2}$-terms in the Lagrangian is reduced to irrelevant constant in the entropy [6], and for this reason we omit these terms. For massless fields there is 
also an infrared divergence in (12). It can be eliminated in the same manner [4] as for the classical Einstein action (4) by subtracting from the effective gravitational functional $W$ (11) additional terms given on a distant spatial boundary $r=r_{0}$. After the subtraction, the action (11) turns out be finite on (5) and includes terms of the order $O\left(r_{0}^{-1}\right)$ that can be neglected in the limit $r_{0} \rightarrow \infty$. We imply that infrared divergences are removed in such a way but do not write the boundary terms in (11) explicitly since their form is also irrelevant for further consideration.

For the functional (11) taken on the space (5) the only free parameter, apart from $\beta$, is the mass $m$ of the hole and as in classical theory we can consider its variation with respect to this parameter. Thus, the extremality condition of $F(\beta)$ can be represented as

$$
\beta-8 \pi m+\left(\frac{\partial W_{Q}}{\partial m}\right)_{\beta}=0
$$

Equation (13) indicates a correction $\partial_{m} W_{Q}$ to the Hawking temperature which can be calculated as follows. Consider the scaling properties of $W_{Q}$ that depends on $m$ through the background metric (5) and on $\beta$ through the boundary conditions. Assuming $W_{Q}$ to be a renormalized action, one can write

$$
\begin{gathered}
W_{Q}\left(\beta, g_{\mu \nu}(m)\right)=W_{Q}\left(\beta \alpha^{-1}, \alpha^{2} g_{\mu \nu}\left(m \alpha^{-1}\right)\right)= \\
W_{Q}\left(\beta \alpha^{-1}, g_{\mu \nu}\left(m \alpha^{-1}\right)\right)+\left(\int T_{\mu}^{\mu} \sqrt{g} d^{4} x-a_{\text {surf }}\left(\beta \beta_{H}^{-1}\right)\right) \log \alpha
\end{gathered}
$$

where $\alpha$ is an arbitrary parameter and $\beta_{H} \equiv 8 \pi m$. The last term in the r. h. s. of (14) appears due to the breaking in $W_{Q}$ of the conformal invariance to be held for classical massless fields. It includes the standard trace anomaly of the renormalized stress tensor $T_{\mu}^{\mu}=-\left(16 \pi^{2}\right)^{-1} a_{2}$ determined by the $a_{2}$-coefficient in the DeWitt-Schwinger proper time expansion [9]. In our case, it is

$$
\int_{0}^{\beta} d \tau \int_{2 m}^{\infty} r^{2} d r \int d \Omega T_{\mu}^{\mu}=\sigma \frac{\beta}{\beta_{H}}
$$

where $\sigma$ is the integral of the trace anomaly at $\beta=\beta_{H}$ that depends on the numbers $N_{s}$ of the fields with the spin $s$ entering in the model [9], [10]

$$
\sigma=\frac{1}{45}\left(-N_{0}-\frac{7}{4} N_{1 / 2}+13 N_{1}+\frac{233}{4} N_{3 / 2}-212 N_{2}\right)
$$


There is also an additional anomalous term $a_{\text {surf }}\left(\beta \beta_{H}^{-1}\right)$ in the transformation law of $W_{Q}$ due to the conical singularities of the background manifold; $a_{\text {surf }}\left(\beta \beta_{H}^{-1}\right)$ is an integral over the horizon surface which has been exactly found for the scalar determinants in [11]. However, the only thing important for us is that this addition disappears at the Hawking temperature

$$
a_{\text {surf }}\left(\beta \beta_{H}^{-1}\right)=0 \quad, \quad \beta=\beta_{H}=8 \pi m
$$

It is suitable to choose $\alpha=m$ and represent (14) as

$$
W_{Q}\left(\beta, g_{\mu \nu}(m)\right)=W_{Q}\left(\beta m^{-1}, g_{\mu \nu}(m=1)\right)+\left(\sigma \beta \beta_{H}^{-1}-a_{\text {surf }}\left(\beta \beta_{H}^{-1}\right)\right) \log m \equiv f\left(\beta m^{-1}, m\right)
$$

This immediately results in the relation

$$
\begin{gathered}
\left(\frac{\partial W_{Q}}{\partial m}\right)_{\beta}=\left(\frac{\partial f}{\partial m}\right)_{\left(\beta m^{-1}\right)}-\frac{\beta}{m}\left(\frac{\partial f}{\partial \beta}\right)_{m}= \\
\frac{1}{m}\left(\sigma \frac{\beta}{\beta_{H}}-a_{\text {surf }}\left(\beta \beta_{H}^{-1}\right)\right)-\frac{\beta}{m}\left(\frac{\partial W_{Q}}{\partial \beta}\right)_{m} .
\end{gathered}
$$

Inserting (19) into condition (13) we have

$$
\beta-8 \pi m-\frac{\beta}{m}\left(\left(\frac{\partial W_{Q}}{\partial \beta}\right)_{m}-\frac{\sigma}{\beta_{H}}\right)-\frac{1}{m} a_{\text {surf }}\left(\beta \beta_{H}^{-1}\right)=0
$$

and $\beta$ can be found from (20) by iteration in the Planck constant $\hbar$ as a series. Thus, taking into account (17), one obtains the expression

$$
\beta=8 \pi\left(m+\left(\frac{\partial W_{Q}}{\partial \beta}\right)_{m}-\frac{\sigma}{8 \pi m}\right)+O\left(\hbar^{2}\right)
$$

The quantity $\partial_{\beta} W_{Q}$ in (21) is the thermal energy of quantum fields associated with the radiation of a hole and it is an unknown functional of the background metric. Fortunately, there is no need to calculate it explicitly so far as equation (21) can be rewritten through the total internal energy of the system

$$
E=\frac{\partial}{\partial \beta}(\beta F)_{m}=m+\left(\frac{\partial W_{Q}}{\partial \beta}\right)_{m} \equiv M .
$$

The constant $M$ can be considered as the effective gravitational mass including the energy of the radiation and, as distinct from the classical mass $m$, it is an observable parameter of the theory. In terms of $M$ and in the first order in $\hbar, \beta$ takes the simple form

$$
\beta=8 \pi\left(M-\frac{\sigma}{8 \pi M}\right)
$$


(replacing $m$ by $M$ in the anomalous term in (21) results in a correction $O\left(\hbar^{2}\right)$ ). Consequently, the one-loop temperature reads

$$
T=T_{H}(M)\left(1+\frac{\sigma}{8 \pi M^{2}}\right)
$$

where $T_{H}(M)=(8 \pi M)^{-1}$ is the classical Hawking temperature defined for a hole with the mass $M$. The one-loop entropy can be recovered from (24) by making use of Clausius's rule

$$
S=\int \frac{d M}{T}=4 \pi M^{2}-\sigma \log M
$$

and it differs from the Bekenstein-Hawking entropy by the logarithmic term. Another way to derive (25) is to use the statistical-mechanical definition (11) of $S$. Equations (24) and (25) represent the main result of this paper. Remarkably that $T$ and $S$ can be found explicitly and coming out is the only new coefficient $\sigma$ of the field species given by equation (16).

The temperature $T$ has been derived from the extremum of the one-loop free energy or, which is the same, of the effective gravitational action $W$, see (11). Although $W$ is a non-trivial functional of the metric, one can expect that it possesses the same property as the Euclidean Einstein action (雨) when quantum effects are weak and has the extrema on the smooth manifolds with the black hole geometry similar to (5). This seems to be a natural assumption because, as was pointed out, non-smooth solutions with conical singularities would correspond to some specific matter distribution concentrated on the horizon surface of a hole. Therefore, in quantum case one can repeat the same arguments given above for the classical action (4) and relate the extremum (13) of $W$ with vanishing of the conical singularity for the Schwarzschild solution deformed by one-loop quantum corrections. After that the temperature (24) should be related with the one-loop surface gravity $k$ as $T=(2 \pi)^{-1} k$ and, hence, one can identify it with the temperature of the Hawking radiation in presence of the back reaction.

Let us discuss these results. As is seen from (24) and (25), in the model in question the difference of $T$ and $S$ from their classical form is completely provided by the conformal anomaly (16). In this context it is interesting to pay attention to the role played by the anomalous trace in two dimensional theory where it determines the flux of the Hawking 
radiation [12]. In four dimensions the anomaly is known to be absent in the models of $N=8$ and $N=4$ supergravity and in the $N=4$ super Yang-Mills theory [10]. Thus, following from (24) and (25) is an interesting consequence that for these models the oneloop corrections can change the mass of the Schwarzschild black hole, whereas the form of the thermodynamics is left the same as in the classical case. In general, the behavior of $T$ and $S$ depends on the sign of $\sigma$. The latter is positive when spins 1 and $3 / 2$ dominate in the theory and then quantum effects accelerate evaporation of the hole by increasing its temperature.

A qualitative difference from the classical black hole thermodynamics appears for the negative anomaly $\sigma<0$, when the scalars, fermions and gravitons prevail. Then, the increase in the hole temperature $T$ slows down. Moreover, in this case, when mass approaches the Planck scales $M \simeq \sqrt{\sigma} M_{\text {Planck, }} T$ reaches a maximum and after that starts to decrease, a hole cools down. Surely, in this domain the one-loop approximation is not reliable and another, probably, nonperturbative treatment is needed. However, if (24) is used for extrapolation to the Planck region, it shows that temperature is null for some small or zero values of $M$, which can be interpreted as the end of evaporation. If this were actually true, the black hole evaporation would finish by a pure vacuum state. This eventually would remove the information loss paradox [1].

Our analysis would be incomplete without comparing equations (24) and (25) with the one-loop quantities derived by taking directly into account the back reaction caused to the Schwarzschild metric by the quantum matter [13], [14]. However, to employ the back-reaction method, one needs the renormalized stress tensor that is known for the Schwarzschild hole in 4-dimensions only in the Page approximation [15]. Nevertheless, there is a qualitative agreement between equation (24) and that reported in [13], [14]. In particular, the maximum of the radiation temperature was also mentioned in [14] for the gravitation dominated matter. It is also worth pointing out that a logarithmic dependence of the one-loop black hole entropy on the mass, similar to (25), has been found out in a number of two-dimensional models, for instance in [16], and has been argued to occur in the membrane approach to the description of black holes [17.

One should remark in conclusion that the reason why the simple expressions for $T$ 
and $S$ have been obtained in our method is that the Schwarzschild metric possesses the only dimensional parameter $m$. Thus, it is interesting to repeat the analysis for more general black hole geometries and massive quantum fields and see how the properties of the considered model can change.

The author is very grateful to Professor Igor Novikov for hospitality at Theoretical Astrophysics Center in Copenhagen and helpful discussions and also thanks Sergey Solodukhin for valuable contacts. This work was partially supported by Russian Foundation for Fundamental Science, grant N 94-02-03665-a.

\section{References}

[1] J.D. Bekenstein, Do we understand black hole entropy?, gr-qc/9409015.

[2] C.G. Callan, S.B. Giddings, J.A. Harvey and A. Strominger, Phys. Rev. D45, R1005 (1992); J.G. Russo, L. Susskind and L. Thorlacius, Phys. Rev. D46, 3444 (1992), Phys. Rev. D47, 533 (1993).

[3] L.D. Landau and E.M. Lifshitz, Statistical Physics (Pergamon Press, London - Paris, 1958).

[4] G.W. Gibbons, S.W. Hawking, Phys. Rev. D15, 2752 (1977); S.W. Hawking in General Relativity, ed. by S.W. Hawking and W. Israel (Cambridge Univ. Press, Cambridge, 1979).

[5] G.H. Hayward and J. Louko, Phys. Rev. D42, 4032 (1990).

[6] D.V. Fursaev and S.N. Solodukhin, On description of the Riemannian geometry in presence of conical singularities, in preparation.

[7] D.V. Fursaev and S.N. Solodukhin, On one-loop renormalization of black hole entropy, preprint E2-94-462, hep-th/9412020.

[8] G.'t Hooft, Nucl. Phys. B256, 727 (1985). 
[9] N.D.Birrell and P.C.W.Davies, Quantum Fields in Curved Space (Cambridge Univ. Press, New York 1982).

[10] S.M. Christensen and M.J. Duff, Phys. Lett. B76, 571 (1978); M.J. Duff, Class. Quantum Grav. 11, 1387 (1994).

[11] J.S. Dowker, Effective actions with fixed points, preprint MUTP/94/14, hepth/9406144.

[12] S.M. Christensen and S.A. Fulling, Phys. Rev. D15, 2088 (1977).

[13] J.W. York, Phys. Rev. D31, 775 (1985).

[14] C.O. Lousto and N. Sanchez, Phys. Lett. 212, 411 (1988).

[15] D.N. Page, Phys. Rev. D25, 1499 (1982).

[16] T.M. Fiola, J. Preskill, A. Strominger and S.P. Trivedy, Black hole thermodynamics and information loss in two dimensions, preprint CALT-68-1918, hep-th/9403137; S.N. Solodukhin, The conical singularity and quantum corrections to entropy of black hole, preprint JINR E2-94-246, Phys. Rev. D to be published.

[17] C.O. Lousto and M. Maggiore, On the energy spectrum of quantum black holes, preprint IFUP-TH 53/94, gr-qc/9411037. 